\title{
Critical adsorption controls translocation of polymer chains through lipid bilayers and permeation of solvent
}

\author{
Jens-Uwe Sommer* and Marco Wernet \\ Leibniz-Institut für Polymerforschung Dresden e.V., Hohe Strasse 6, \\ 01069 Dresden, Germany and Technische Universität Dresden, \\ Institute of Theoretical Physics, 01069 Dresden, Germany \\ Vladimir A. Baulin $\ddagger$ \\ ICREA, 23 Passeig Lluis Companys, Barcelona 08010, \\ Spain and Departament d'Enginyeria Quimica, \\ Universitat Rovira i Virgili, 26 Av. dels Paisos, \\ Catalans, Tarragona 43007, Spain
}

\begin{abstract}
Monte Carlo simulations using an explicit solvent model indicate a new pathway for translocation of a polymer chain through a lipid bilayer. We consider a polymer chain composed of repeat units with a given hydrophobicity and a coarse-grained model of a lipid bilayer in the self-organized liquid state. By varying the degree of hydrophobicity the chain undergoes an adsorption transition with respect to the lipid bilayer. Close to the transition point, at a properly balanced hydrophobicity of the chain, the membrane becomes transparent with respect to the chain. At the same time the solvent permeability of the bilayer is strongly increased in the region close to adsorbed chain. Our results indicate that the critical point of adsorption of the polymer chain interacting with the fluctuating lipid bilayer could play a key role for the translocation of molecules though biological membranes.
\end{abstract}


Lipid bilayers formed by self-organization of amphiphilic molecules form the natural border of living cells, thus protecting the interior of the cell from the environment, but also allowing for exchange of substances and information between the cell and its surroundings. A challenging problem is to understand the translocation of biopolymers through lipid bilayers. In particular it would be desirable to know under which conditions polymers can cross the cell membrane which otherwise forms a strong barrier against exchange of larger molecules. Some anti-microbial peptides [1], cell-penetrating peptides [2] and drugdelivery polymers [3] have been shown to penetrate through phospholipid bilayers. Despite considerable effort devoted to the problem, physical principles underlying their membrane activity and translocation behavior are not well understood. The suggested mechanisms of interaction of biopolymers with bilayers [4] imply formation of static structures in the membranes such as stable pores. However, the formation of static well-ordered structures by polymers without a secondary structure is questionable, and their translocation through membranes [3, 5] may indicate the existence of other translocation mechanisms. Recent studies [3] have shown that at certain conditions amphiphilic copolymers can translocate through membranes and simultaneously enhance permeability of membranes for low molecular components without evidence of formation of static pores or other structures. Such ability to facilitate the permeation of membranes has important applications in red blood cells preservation [3], trans-membrane drug delivery [4], enhancement of intestinal wall for drugs [6] and gene delivery [7].

Understanding these mechanisms of interaction of polymers with bilayers is of particular importance for development of efficient delivery vectors which are able to transport drugs or foreign DNA into the cell [8-10]. On the other hand, there are indications that biopolymers interacting with cell membranes might significantly increase permeability with respect to small molecules such as trehalose depending on the amount of charged side groups of the polymer [3].

In this work we explore a simple model-system where the lipid bilayer is formed by coarse grained and flexible amphiphilic molecules using an explicit solvent model to prevent artifacts due to strong hydrophobic interactions, but also to be able to consider the permeability of the self-organized membrane with respect to the solvent. Polymer chains interact with the membrane via an effective hydrophobicity of the monomers which should mimic the effect of a hetero-polymer having both hydrophilic and hydrophobic components. Charge effects 


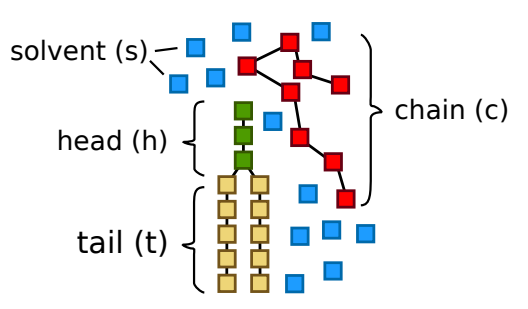

(a)

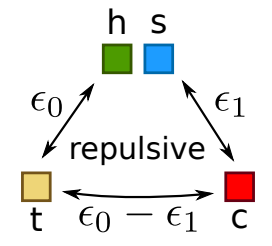

(b)

Figure 1: Implementation of the bond fluctuation model. (a) coarse grained representation of lipids, explicit solvent and polymer chains as used in our BFM simulations. (b) repulsive interactions between the four components as defined in (a) and their associated energy constants $\epsilon_{0}$ and $\epsilon_{1}$.

will be disregarded in our work focusing on short-range interactions between the monomers of the chains and the monomers of the lipid-molecules. These simplifications allow for the understanding of a fundamental relation between the hydrophobic interactions between polymers and lipids and the translocation and permeability barrier of the lipid-bilayer. In particular we will show that lipid-bilayers become transparent for the polymer chains if the effective hydrophobic interactions drives the chains near the adsorption threshold with respect to the membrane. Deviating from this adsorption threshold only slightly the chain is either rejected from the membrane, or trapped inside the hydrophobic core. We also demonstrate that close to the adsorption threshold the permeability of the membrane with respect to the solvent molecules in the environment of the chain is strongly increased. After presenting briefly some details of the simulation method we will report our results.

In order to simulate lipid bilayers and their interaction with linear chains we use the bond fluctuation method (BFM) [11, 12], using a short-range interaction model between the different species. The model is well established to simulate various polymer systems, details of our implementation of the interaction model can be found in Ref. [13]. In this work we consider an explicit solvent model which avoids freezing effects and related artifacts due to strong attractive interactions imposed by solvent-free models [14, 15]. The essential details of the model are illustrated in Fig,1. We simulate two-tailed lipids with five monomers per tail (t) and three monomers per head (h) as well as single polymer chains (c) of various chain lengths, $L_{c}$, see Fig. 1(a). Furthermore, an explicit solvent (s) represented by unconnected BFM-units is taken into account. The simulation box is filled up by solvent reaching a volume occupation of 0.5 , which corresponds to a dense system in the framework of the BFM. Within 
the framework of the simulation model packing effects of the lipids are taken into account. Since we use local monomer moves only the algorithm mimics a proper diffusional dynamics of polymers, lipids and solvent.

The hydrophobic effect is modeled as a short-range repulsive interaction (energy penalty for pair contacts) between monomers of different hydrophobicity. Each of the 24 next nearest lattice sites of a tagged BFM-monomer of type $A$ is associated with an energy penalty, $\epsilon_{A B}$, if it is occupied by an interacting monomer of type $B$ [16]. The resulting internal energy of the tagged monomer rules the elementary Metropolis step. In a four component system $(\mathrm{t}, \mathrm{h}, \mathrm{c}, \mathrm{s})$ in the most general case six interaction constants can be fixed. We used a simplified interaction model according to Fig. 1, where only two independent interaction energies, $\epsilon_{0}$ and $\epsilon_{1}$ are considered according to

$$
\begin{aligned}
\epsilon_{0} & =\epsilon_{t, h}=\epsilon_{t, s} \\
\epsilon_{1} & =\epsilon_{h, c}=\epsilon_{s, c}=\epsilon_{0}-\epsilon_{t, c} \\
\epsilon_{h, s} & =0
\end{aligned}
$$

Hence, we assume that solvent (s) and heads (h) are indistinguishable with respect to their interactions with other (hydrophobic) species. Applying the above model to random starting configurations of lipids in solvent (without chains), self-assembled lipid bilayers in a planaror vesicular shape were obtained depending on boundary conditions and lipid volume fraction. In the following we consider planar bilayers consisting of 300 lipids in a cubic box of size 64 with periodic boundary conditions in all directions. The starting configuration of the bilayer has been chosen such that the self-organized membrane spreads over the periodic boundaries in $x$ - and $y$-direction and shows a stable orientation perpendicular to the $z$-axis. Therefore, the $(x, y)$ - plane of the lattice is subsequently used as a fixed reference-plane for the bilayer. We have chosen $\epsilon_{0}=0.8$ (units of $k_{B} T$ ) which leads to the formation of stable bilayers. To characterize the extension of the bilayer, we calculated the center of mass $\bar{z}=\langle z\rangle$ and the variance $\sigma_{z}^{2}=\left\langle z^{2}\right\rangle-\bar{z}^{2}$ of the $z$-positions of all tail monomers during our simulations. We define the boundaries of the bilayer by $z_{ \pm}=\bar{z} \pm 2 \sigma_{z}$. We added single chains of various chain length, $L_{c}$, to the simulation box with an effective hydrophobicity, $\epsilon_{1}$. Typical snapshots for $L_{c}=64$ are shown in Fig. 2 ,

In the following we show simulation results under variation of $\epsilon_{1}$ at constant $\epsilon_{0}=0.8$. 


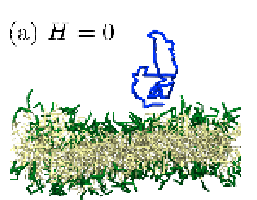

$3.34 \times 11 \times \mathrm{MCS}$

(b) $H=0.68$

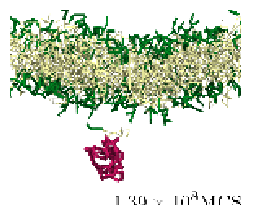

$1.14 \times 101^{1} \mathrm{MCS}$

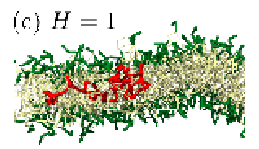

$10 \% \times 10^{3} \mathrm{MOS}$

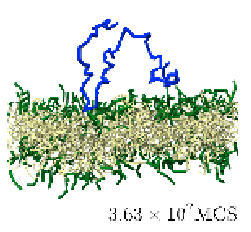

$3.63 \times 10^{\circ} \mathrm{YCS}$

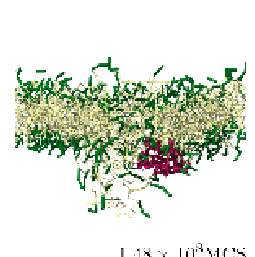

$1.1 \times 1.0^{3} \mathrm{Mes}$

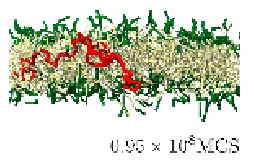

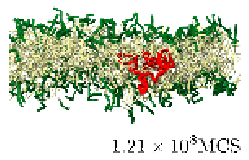

4

L.fit $\times 10^{\mathrm{R}} \mathrm{MC}$

$1.21 \times 10^{3} \mathrm{TC}$

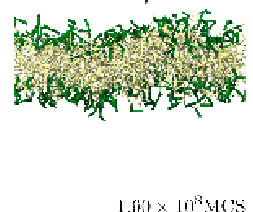

Figure 2: Snapshots of BFM-simulations (see also Fig. 1 and Eq.(1)) of lipid bilayers and linear polymer chains $\left(L_{C}=64\right)$ with various relative hydrophobicity, $H$, taken at given simulation times.

We define the relative hydrophobicity of the chain according to

$$
H=\epsilon_{1} / \epsilon_{0}
$$

As can be seen in Fig. 2(a), for $H=0$ the (hydrophilic) chain is rejected by the lipid bilayer and its conformations correspond to a self-avoiding walk in good solvent. For $H=1$ (hydrophobic) the chain is collapsed in the solvent but is strongly attracted and eventually absorbed by the bilayer's core. At intermediate values close to $H=0.68$ (partial hydrophobic) the chain can be found in the solvent phase as well as penetrating the bilayer . Note that the chain is collapsed at this value of $H$ and explicit solvent simulation enables a non-frozen liquid-like dynamics of the chain.

By increasing the relative hydrophobicity the bilayer changes its energetic characteristics from a potential barrier for $H=0$ into a potential trap for $H=1$ with respect to the chain. This resembles an adsorption scenario of a chain at a penetrable potential well.

In general, one expects a characteristic value of $H_{A}$ where the chain is just at the transition to the adsorbed state. In order to characterize the transition point in our model we have calculated the fluctuation of the contact energy $U_{\mathrm{t}, \mathrm{c}}$, (heat capacity) between tail monomers and chain monomers as shown in Fig. 3(a). At the transition point the fluctuations display a 


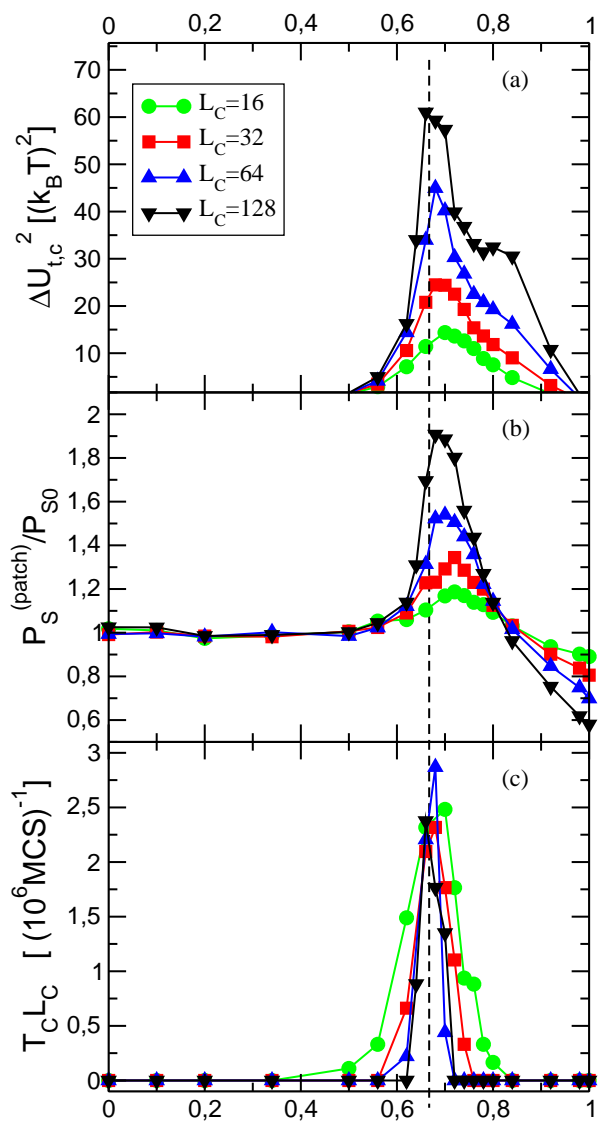

Figure 3: (a) Fluctuation of the internal energy, $\Delta U_{\mathrm{t}, \mathrm{c}}{ }^{2}=\left\langle U_{\mathrm{t}, \mathrm{c}}^{2}\right\rangle-\left\langle U_{\mathrm{t}, \mathrm{c}}\right\rangle^{2}$, corresponding to nearest neighbor-contacts between tail-monomers in a lipid bilayer and monomers of a linear chain of length $L_{c}$ depending on relative hydrophobicity, $H$, see Eq. (11). The extrapolated adsorption transition, $H_{A}=0.667 \pm 0.005$, is indicated by the vertical dashed line.(b) Simulation results for the solvent-permeability, $P_{S}^{(\text {patch })}$, of a circular membrane patch (projected radius $\approx 15$, see text) around the projected center of mass of a polymer chain depending on the relative hydrophobicity $H$. The results are normalized to the permeability for an membrane not interacting with polymer chains (corresponds practically to $H=0$ ), $P_{S, 0}$. (c) Simulation result for the rescaled frequency of translocations, $T_{C}$, of a polymer chain of length $L_{c}$ through a lipid bilayer depending on relative hydrophobicity of the chain.

peak which should approach the critical point of adsorption for $L_{c} \rightarrow \infty$ [17]. For finite chains adsorption corresponds to a crossover scenario where the effective adsorption threshold is increased for decreasing chain length according to $\left(H-H_{A}\right) / H_{A} \sim N^{-\phi}$, where $\phi$ denotes the crossover-exponent for polymer adsorption, whose values depend on the problem at 
hand [17, 18]. Our results suggest that the adsorption transition is close to $H_{A} \simeq 0.667 \pm$ 0.005. We note that a value of $H_{A}>0.5$ indicates a higher chemical potential of the bilayer's core with respect to the solvent (higher density, perturbation of the lipid conformation and local fluctuations of the bilayer due to embedding of the chain or chain parts).

Let us consider the frequency of permeations (translocations) of molecules of species $\alpha$ through the model bilayer,

$$
T_{\alpha}=\frac{n_{\alpha}}{\Delta t} \quad \text { and } P_{\alpha}=\frac{T_{\alpha}}{\mathcal{A}}
$$

where $n_{\alpha}$ is the number of permeation events and $\Delta t$ is the simulation time in units $10^{7}$ Monte Carlo steps (MCS). The permeability, $P_{\alpha}$, of the membrane with respect to species $\alpha$ is proportional to the translocation frequency $\mathrm{T}_{\alpha}$ in the case of a given concentration difference on both sides of the membrane. For simplicity, let us here define the permeability as a translocation frequency per surface area where the surface area, $\mathcal{A}$, is given by the projection of the membrane to the $(x, y)$-plane of the lattice.

We detected permeation events both of solvent molecules as well as of the linear chain using the trajectories of their centers of mass with a time resolution of 100 MCS. The zcomponents of the trajectories have been analyzed with respect to their crossing points with two thresholds, $l_{ \pm}$, on both sides of the bilayer center, $\bar{z}$. If a molecule passed successively through both planes defined by $l_{+}$and $l_{-}$we counted one permeation event for the respective molecule species. For the solvent molecules we define the two thresholds, $l_{ \pm}$, to be identical to the boundaries of the bilayer, $l_{ \pm}^{(S)}=z_{ \pm}$. For the polymer chain we fixed the thresholds to $l_{ \pm}^{(C)}=\bar{z} \pm 23$, where we concluded from the center of mass distribution of the longest chains, $L_{C}=128$, that they are well separated from the bilayer.

The results for the frequency of translocations of the chain, $T_{C}(H)$, are displayed in Fig. 3(c). Here, we have rescaled the frequency of translocations with the center of mass diffusivity of the free chain which is $\sim 1 / L_{C}$. Translocation of the chain is clearly shown close to the adsorption transition. We note that at $H_{0}=1 / 2$ the lipid tails are equally repulsive as the solvent with respect to the chains. However, in the self-assembled state the tails are stretched and partially ordered which imposes a Free energy effort to insert the chain into the bilayer at $H_{0}$ similar to the case of a polymer brush [19, 20]. Let us denote this Free Energy barrier by $\Delta F_{\text {ins }}=N \Delta \mu$. In order to compensate this barrier an effective attraction strength of $\epsilon=\chi_{0} \epsilon_{0} \Delta$ with $\Delta=\left(H-H_{0}\right) / H_{0}$ is necessary. Here, $\chi_{0}$ accounts for the average number of monomers interacting with a given chain monomer. For $\mu=\epsilon$ the free energy 
barrier vanishes. On the other hand, surface adsorption of the chain can occur, because for monomers directly in contact with the bilayer's core an effective attraction potential with a depth of $-\epsilon / \chi_{0}$ is forming on both sides of the core. The chain can be localized at this potential trap with a limited penetration into the core. A closer analysis for the case of an ideal chain reveals an adsorption threshold [21] which very close to $\mu=\epsilon$. This can explain the coincidence between the critical point of adsorption and the translocation of the chain. However, we note that the ideal chain model might not be fully appropriate here since the chain is in a collapsed state at the transition point.

An interesting measure for perturbations of the bilayer due to an adsorbing polymer chain is the permeability, $P_{S}$, of the bilayer with respect to the solvent molecules. For each solvent permeation event we have recorded the distance between the position of the solvent unit and the center of mass of the chain projected into the reference plane of the bilayer, $\vec{d}$, taken in the moment of the beginning of the translocation event. In Fig. 3(b) we show simulation results for the local permeability $P_{S}^{(\text {patch })}$ through a circular membrane patch of (projected) radius $|\vec{d}|<15$. This defines a patch on the membrane that follows the center of mass of the chain as a "shadow". For chains length $L_{C}=128$ the local permeability $P_{S}^{(\text {patch })}$ increases up to a factor of almost two with respect to the unperturbed bilayer if the chain is close the adsorption transition. It is notable that for strong hydrophobicity of the chain, $H \rightarrow 1$, the permeability decreases up to a factor of almost two, indicating a stabilizing effect of the embedded chain. The peak of the solvent permeability correlates with the effective adsorption transition of the chain for different chain lengths. 

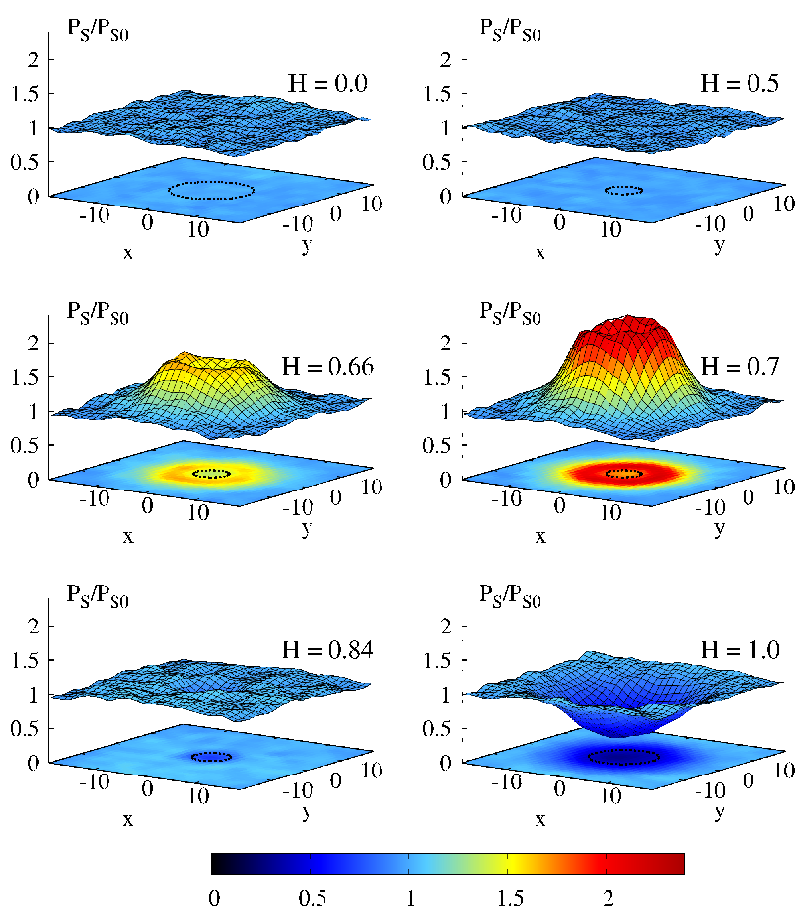

Figure 4: Local solvent-permeability $P_{S}$ of a lipid bilayer as a function of the Cartesian distance vector $(x, y)$ in the membrane's reference plane from the projected center of mass position of a single polymer chain. We show results for various degrees of the relative hydrophobicity, $H$, normalized to the average permeability $P_{S 0}$ in the case of $H=0$.

In Fig. 4 we show the local permeability $P_{S}(\vec{d})$ for various hydrophobicities $H$ as a function of the projected distance vector between entering points and chain, $\vec{d}$. The permeability of the solvent is only increased in the immediate environment of the adsorbing chain near $H_{A}$. Note that close to the center of the chain solvent permeability is partially screened by the polymer globule.

To conclude our results we have shown that for a simplified model of the lipid bilayer the point of adsorption of the chain controls both the solvent permeability and the chain translocation through the membrane. This can be explained by assuming the membrane core as a potential well for the polymer chain which becomes transparent if the relative hydrophobicity of the monomers is balanced in such a way that both the solvent and the hydrophobic core of the membrane are equally poor environments for the chain. The translocation maximum and the point of adsorption indicate a threshold for insertion of chain monomers into the bilayer core. Perturbation of the membrane properties due to chain adsorption correlates with enhanced solvent-permeability of the membrane close to the adsorption point. In 
biological systems the polymers are usually represented by amphiphilic copolymers, such as polypeptides, with a possibly disordered arrangement of both hydrophilic and hydrophobic monomers. Hydrophobic compensation and adsorption discussed in our work then depends on the ratio of hydrophilic and hydrophobic monomers. Thus, a common feature of membrane-crossing polymers can be the proper balance of the effective hydrophobicity.

* Electronic address: sommer@ipfdd.de

$\dagger$ Electronic address: werner-marco@ipfdd.de

$\ddagger$ Electronic address: vladimir.baulin@urv.cat

[1] K. A. Brogden, Nature Reviews 3, 238 (2005).

[2] N. Nehkotiaeva, A. Elmquist, G. Kuttuva-Rajarao, M. Hallbrink, U. Langel, and L. Good, FASEB Journal 18, 394 (2004).

[3] A. L. Lynch, R.-J. Chen, P. J. Dominowski, E. Y. Shalaev, R. J. Yancey, and N. K. H. Slater, Biomaterials 31, 6096 (2010).

[4] F. Mathot, A. Schanck, F. V. Bambeke, A. Arien, M. Noppe, M. Brewster, and V. Preat, Journal of Controlled Release 120, 79 (2007).

[5] T. Goda, Y. Goto, and K. Ishihara, Biomaterials 31, 2380 (2010).

[6] S. Muranashi, Crit. Rev. Ther. Drug Carrier Syst. 7, 1 (1990).

[7] D. W. Pack, A. S. Hoffman, S. Pun, and P. S. Stayton, Nature Rev. Drug. Disc. 4, 581 (2005).

[8] A. Prochiantz, Adv. Drug. Deliv. Rev. 60, 448 (2008).

[9] A. Prochiantz, Nature Methods 2, 119 (2007).

[10] M. Zorko and U. Langel, Adv. Drug. Deliv. Rev. 57, 529 (2005).

[11] I. Carmesin and K. Kremer, Macromolecules 21, 2819 (1988).

[12] H. Deutsch and K. Binder, JCP 94 (1990).

[13] M. Werner and J.-U. Sommer, Eur. Phys. J. E 31, 383 (2010).

[14] G. Reddy and A. Yethiraj, Macromolecules 39, 8536 (2006).

[15] J. Luettmer-Strathmann, F. Rampf, W. Paul, and K. Binder, J. Chem. Phys. 128, 064903 (2008).

[16] A. Hoffmann, J.-U. Sommer, and A. Blumen, J. Chem. Phys. 106, 6709 (1997).

[17] E. Eisenriegler, K. Kremer, and K. Binder, J. Chem. Phys. 77, 6296 (1982). 
[18] E. Bouchaud and M. Daoud, J. Physique Paris 48, 1991 (1987).

[19] F. J. Solis and H. Tang, Macromolecules 29, 7953 (1996).

[20] A. Halperin, G. Fragneto, A. Schollier, and M. Sferrazza, Langmuir 23, 10603 (2007).

[21] S. Stepanow, U. Bauerschäfer, and J.-U. Sommer, Phys. Rev. E 54, 3899 (1996). 